\section{Gene therapy for cystic fibrosis}

To the editor - The article by Caplen et al. in your first issue ${ }^{1}$ provided a welcome progress report on attempts to develop gene therapy for patients with cystic fibrosis. Its cautious tone contrasted sharply with the exaggerated prospects for gene therapy claimed in the News and Views section of the same issue ${ }^{2}$. After recounting the extraordinary progress towards gene therapy which has occurred since the discovery of the CFTR gene in 1989, and after outlining some of the major problems which remain, the author made what I believe to be unwarranted assertions. Looking into a "Crystal ball" he states that "there is no question that in vivo gene therapy for CF will be reality" finding the arguments for the continued development of in vivo gene therapy "far too compelling". He went on to conclude that "with luck and hard work it will ... result in a cure for cystic fibrosis".

As currently being pursued, gene therapy will not cure cystic fibrosis. Cystic fibrosis is a systemic disease affecting the pancreas, intestines, liver and reproductive system as well as the lungs. Even if the inhaled gene were to be incorporated into the genome - an outcome which is currently prohibited by international ethical convention - it would not reverse the damage to the pancreas or male genital tract. Nor is it likely that it would materially improve function in the lungs of thousands of patients with CF, where much damage has already occurred. We simply do not know what to expect in terms of the duration of effect of the frequency with which inhalations may need to be repeated, and there is considerable uncertainty about the exact respiratory epithelial cells which should be targeted.

The only form of gene therapy which might be expected to "cure" cystic fibrosis with its many manifestations would be insertion of the gene during fetal life, and in this sense I agree with Dr. Crystal that gene therapy may some day be a reality. In the meantime, we should explore other promising avenues and build on the understanding of CFTR function which has been developing even more rapidly than attempts at gene therapy. For example, the observation that in cells affected by the commonest mutation, $\Delta \mathrm{F} 508$, maturational arrest of CFTR leads to failure of its incorporation into the apical membrane $^{3}$ led to the exciting discovery that reducing the temperature of CF cells in culture promotes improved delivery of CFTR to the cell membrane, where it functions reasonably well ${ }^{4}$. This raises the possibility that a pharmacological agent might be found that would have the same effect as cooling - and obviate the many problems of gene therapy.

The treatment of cystic fibrosis has seen many false dawns, but the prognosis has steadily improved by the practice of good medicine. It is unrealistic to think that new treatments will have a major impact on life expectancy in the foreseeable future, and I recently read with a mixture of sorrow and frustration a newspaper report of an adult CF patient who was anticipating that "gene therapy" would free her from the need to carry out daily physiotherapy. It is in order to counter such unjustified euphoria and to help patients live with the day-to-day reality of their diseases that I ask your readers to take a balanced and responsible view of the prospects for control of cystic fibrosis.

1. Caplen, N.J. et al. Liposome-mediated CFTR gene transfer to the nasal epithelium of patients with cystic fibrosis. Nature Med. 1, 39-46 (1995).

2. Crystal, R.G. The gene as the drug. Nature Med.1, 15-17 (1995).

3. Purchelle, E. et al. Differential localization of the cystic fibrosis transmembrane conductance regulator in normal and cystic fibrosis airway epithelium. Am. J. respir. Cell molec. Biol. 7, 485-491 (1992).

4. Denning, G.M. et al. Processing of mutant cystic fibrosis transmembrane conductance regulator is temperature-sensitive. Nature 358, 761-764 (1992).

\section{J.A. DODGE}

Queen's University of Belfast

Nuffield Department of Child Health

Institute of Clinical Sciences

Belfast, United Kingdom

\section{IL-6 release and air- way administration of human CFTR cDNA adenovirus vector}

To the editor - The most direct strategy for treating the respiratory manifestations of cystic fibrosis (CF) is in vivo gene therapy, in which the normal human CF transmembrane conductance regulator (CFTR) cDNA is transferred to the airway epithelium, where it can express the CFTR protein and correct the deficiency in cAMP-mediated $\mathrm{Cl}^{-}$secretion in the airway epithelial cells, and thus the chronic airway infection and inflammation that characterizes this disorder ${ }^{1-3}$. The CFTR cDNA can be efficiently transferred to the airway epithelium in vivo using a replication-deficient recombinant adenovirus (Ad) vector ${ }^{1}$. Following extensive safety studies in rodents and non-human primates ${ }^{2-4}$, we initiated human gene therapy for the respiratory manifestations of $C F$ in April 1993. Data from the first four individuals showed that an Ad vector will transfer and express the CFTR CDNA in the airway epithelium in CF (ref. 2). Several groups are now evaluating this strategy in humans $\mathrm{s}^{2,3,5-8}$.

Theoretically, there are several safety issues that are relevant to this therapeutic strategy ${ }^{1-3}$. Most of the issues relating to the vector have turned out to be of minimal concern in humans, as we found no acute immune reactions, no shedding and no in vivo replication ${ }^{2}$. However, in one individual, there was a systemic and local syndrome following administration of $2 \times 10^{9}$ plaque-forming units (pfu) of the AdCFTR vector via a fibre-optic bronchoscope to one lobe of one lung. This syndrome, consisting of headache, fatigue, fever, tachycardia, dyspnea, hypotension and evidence of lung consolidation by physical examination, chest X-ray and lung function testing, resolved spontaneously. Subsequent analysis suggested the large volume of fluid $(20 \mathrm{ml})$ used to administer the vector enabled the vector to reach the alveoli, where it triggered the release of cytokines ${ }^{2}$. In this regard, serum samples taken after administration of the vector showed high (75 $\mathrm{pg} \mathrm{ml}^{-1}$ ) levels of interleukin-6 (IL-6), a cytokine known to induce many of the signs and symptoms observed in this individual ${ }^{9,10}$. By reducing the volume and dose, this syndrome has not been observed in the subsequent six individuals receiving the AdCFTR vector.

Induction of this syndrome in humans was a surprise, as extensive preclinical studies in rodents and nonhuman primates with doses $10^{3}$-fold higher of the same clinical lot of the vector, and with volumes that enabled the vector to reach the alveoli, showed dose-dependent inflammation in the lung, but no other evidence of local or systemic toxicity ${ }^{3,4}$.

The study population included nine CF individuals (six males, three females; $26 \pm 1$ year), all with moderate lung disease $^{2,3}$. Most (7/9) were $\Delta$ F508 homozy- 